\title{
SURFACE PROPERTIES OF CERAMIC HYDROXYAPATITE ELECTRETS ${ }^{\#}$
}

\author{
Katsuyuki Mukogawa ${ }^{1,2^{*}}$, Norio Wada ${ }^{1}$, Naohiro Horiuchi ${ }^{1}$, Miho Nakamura ${ }^{1}$, \\ Akiko Nagai ${ }^{1}$, Toshinori Okura ${ }^{2}$ and Kimihiro Yamashita ${ }^{1}$ \\ (*Corresponding author: bm11057@ns.kogakuin.ac.jp) \\ ${ }^{1}$ Institute of Biomaterials and Bioengineering, Tokyo Medical and Dental University, \\ 2-3-10 Kanda-Surugadai, Chiyoda, Tokyo 101-0062 Japan. \\ ${ }^{2}$ Major of Applied Chemistry and Chemical Engineering, Kogakuin University, \\ 2665-1 Nakanomachi, Hachiouji, Tokyo 192-0015 Japan
}

Keywords: Hydroxyapatite, Polarization, Electret, Surface electric fields

Abstract : Hydroxyapatite (HAp) is the principal inorganic ingredient of human teeth and bone, and well-known as an excellent biocompatible material. HAp has been found to be polarized with an external electric field at a high temperature. Electrets are defined as the solids with functions that quasi-permanent surface charges. However, fundamental characteristics of electric fields due to HAp electrets have never been reported. Hence, this purpose is investigation of the properties of the surface electric fields of HAp bioceramic electrets; the surface potentials due to overlapping plural electrets with different electric potentials and signs; and a screening effect on electric fields with electrets due to a conductor and a dielectric. Polarized charges of the HAp electret have generated an electric field around the electret. The principle of superposition has held for the surface potentials of plural electrets. The screening effect by a conductor or a dielectric has been observed.

(Received January 20, 2012; Accepted March 8, 2012)

Hydroxyapatite (HAp) ceramics have been used as implant materials, because of the crystal structural and compositional analogous with the bone ${ }^{(1)}$. We have studied electric properties of polarized ceramics (electrets). We indicated that the polarization of HAp ascribes to the transportation of the protons in the $\mathrm{OH}$ - columnar structure ${ }^{(2)}$. However, Fundamental characteristics of electric fields due to electrets have never been presented on polymer and ceramic electrets.

Hence, the purpose of this study was to study properties of electric fields due to electrets; the surface potentials due to overlapping plural electrets with different electric potentials and signs, and a screening effect on electric fields due to electrets.

The HAp powders were calcined at $800{ }^{\circ} \mathrm{C}$ for $3 \mathrm{~h}$. and were pressed at $120 \mathrm{MPa}$ in to pellets $(13 \mathrm{~mm}$ diameter and $1.0 \mathrm{~mm}$ thickness $)^{(3)}$. The dense HAp pellets were sintered in a saturated water vapor atmosphere $1250{ }^{\circ} \mathrm{C}$ for $2 \mathrm{~h}$. The HAp pellets that have relative densities above $95 \%$ were used for polling. The pellets sandwiched between platinum plates were polarized in a direct current (DC) field for 1 hour in air. The polarized temperatures were 200, 300 and $400{ }^{\circ} \mathrm{C}$, respectively, and a DC electric field was $5 \mathrm{kVcm}^{-1}$. To avoid any relaxation of the polarization, the electric field was maintained until furnace cooled to room temperature. The polarization state was analyzed by a thermally stimulated depolarization current (TSDC) measurement. The TSDC measurements were carried out in air from RT to $600{ }^{\circ} \mathrm{C}$ at heating rate of $5.0{ }^{\circ} \mathrm{Cmin}^{-1}$. The charge storage (Q) was estimated by integration of the current density of the TSDC curve. A homemade Kelvin probe apparatus was used to measure the surface electric fields (V) of the electrets. Before the Kelvin probe and TSDC measurements, static charges on HAp electrets were eliminate by short circuit.

As to the surface potentials due to overlapping plural electrets with different electric potentials and signs, two HAp electrets were overlapped each other and its surface potential was measured with the Kelvin probe apparatus.

As to a screening effect on electric fields due to electrets, at first, the HAp electret was inserted into two aluminum sheets connected by copper wires and its surface potential was measured with the Kelvin probe apparatus. At next, the wires were cut and the surface potential of the electret was measured with the Kelvin probe apparatus. A screening effect attendant on a paper shell was examined in much the same way as the case of a metal shell. 
The TSDC spectrum for the electret with $\mathrm{Q}=4.3 \mu \mathrm{Ccm}^{-2}$ and $\mathrm{V}=35.5 \mathrm{~V}$ are shown in Fig. 1 by way of example. All of the TSDC curves of the electrets increased at approximately $200{ }^{\circ} \mathrm{C}$ and reached maximum points and then gradually declined, indicating that HAp pellet can be polarized electrically and become electrets. Kelvin probe measurements also revealed that the state of polarization is maintained. These measurements indicate that polarized charges of the HAp electret generate an electric field around the electret.

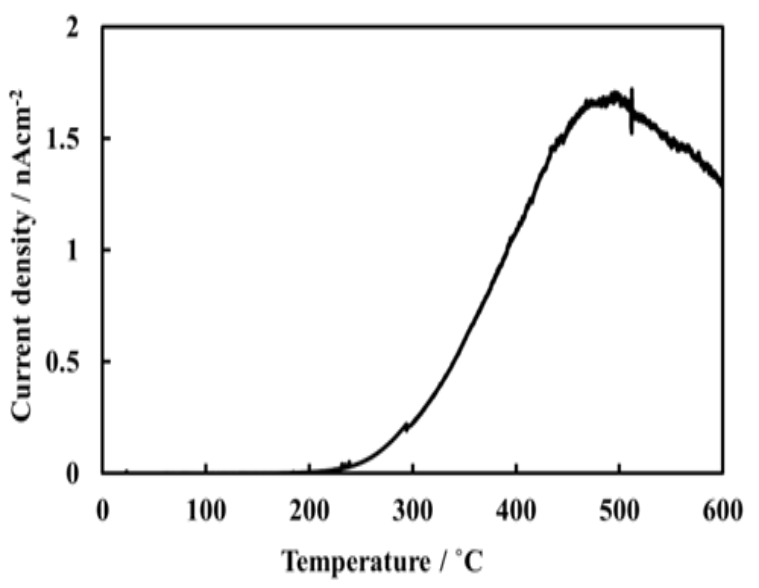

Fig. 1. TSDC spectrum of HAp electret prepared at $400{ }^{\circ} \mathrm{C}$ for $1 \mathrm{~h}$ under $5 \mathrm{kVcm}^{-1}$

The surface potentials of plural electrets, which were obtained according to the addition or subtraction depending on the signs of surfaces, are shown in Fig. 2. The surfaces of the electret with the negative and positive charges were labeled at the $\mathrm{N}$ and P-surfaces, respectively. The close agreement between observed and calculated values was obtained. The result indicated that the electric potentials in given by the algebraic sum of the surface potentials under the condutions that the electrets are averaged in series. This fact indicates that the principle of superposition holds for the electric fields due to HAp electrets.

The screening effects on an electric field with the electrets are illustrated in Fig. 3. The surface potential of polarized HAp was $0 \mathrm{~V}$ under the electret inserted into two aluminum sheets. When the wires are cut, the surface potential improved in magnitude immediately. In the case of paper sheets, the surface potential reduced to half. The screening effect due to a conductor and a dielectric was observed on the electric fields with electrets.

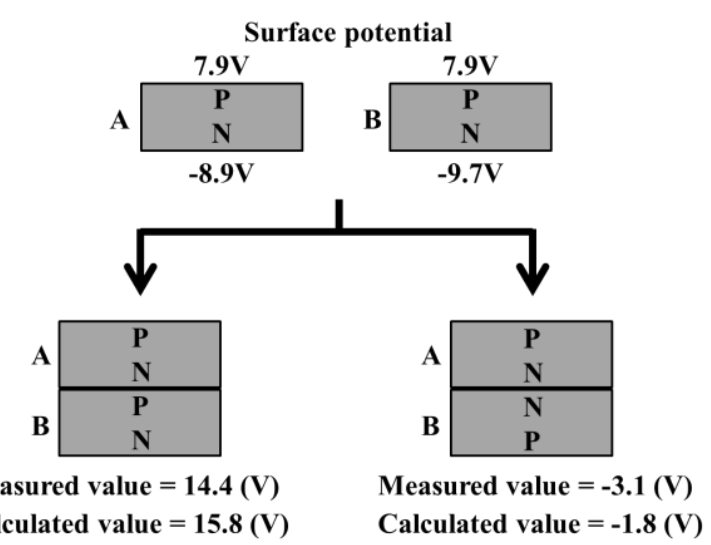

Fig.2. The surface potentials due to overlapping plural electrets with different electric potentials and signs
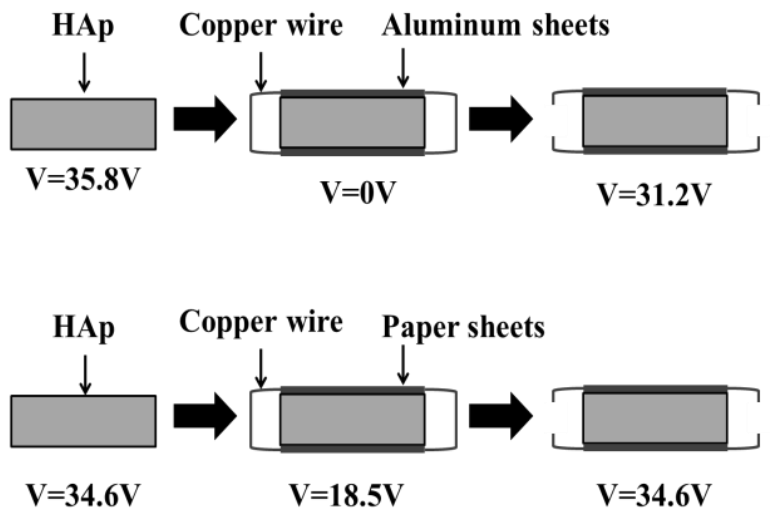

Fig. 3. The screening effects due to paper sheets and aluminum sheets

\section{REFERENCE}

1. S. Nakamura, H. Takeda and K. Yamashita. J. Appl. Phys., 89, 5386-5392 (2001).

2. K. Yamashita, N. Oikawa, and T. Umegaki, Chem. Mater., 8, 2697-2700 (1996).

3. Y. Tanaka, T. Iwasaki, M. Nakamura, A. Nagai, K. Katayama and K. Yamashita, J. Appl. Phys., 107, 14107-14116 (2010).

\#Part of this paper was presented at the meeting The 11th Asian BioCeramics Symposium, which was held in Tsukuba, Japan 2011. 